\title{
THE STUDY OF MULTIDRUG-RESISTANCE IN NEONATAL INTENSIVE CARE UNIT AT THE CENTRAL JAVA HOSPITAL
}

\author{
IKA PUSPITA SARI ${ }^{1 *}$, TITIK NURYASTUTI², DJOKO WAHYONO ${ }^{1}$
}

${ }^{1}$ Department of Pharmacology \& Clinical Pharmacy, Faculty of Pharmacy, Universitas Gadjah Mada, Indonesia. ${ }^{2}$ Department of Clinical Microbiology, Faculty of Medicine, Universitas Gadjah Mada, Indonesia. Email: ika.puspitasari@gmail.com

Received: 2 November 2016, Revised and Accepted: 24 January 2017

\section{ABSTRACT}

Objective: Multidrug-resistance (MDR) is defined as an acquired non-susceptibility to at least one agent in three or more antimicrobial categories. MDR can be caused by several factors, including the misuse of antibiotics. Resistance to antibiotics still poses a global challenge, especially in Indonesia. This study aimed to identify patterns of MDR in neonatal intensive care unit (NICU) at the Central Java Hospital, during the period of January 2014 to December 2015.

Methods: The study was conducted using a descriptive retrospective design. The research population comprised 225 patients. The patient inclusion criteria were neonatal patients treated in NICU ward with infection diagnosis. All patients had culture and sensitivity examinations on their bloods. The culture and sensitivity examinations were performed by microbiology clinicians.

Results: The most common infection type was sepsis (60\%). The most common bacteria found in the blood specimen of patients in the NICU ward was Gram-negative bacteria with a 72\% rate; the other was Gram-positive bacteria. Bacteria which infected patients include Klebsiella pneumoniae ssp. pneumoniae, Pseudomonas aeruginosa, Burkholderia cepacia, Acinetobacter baumannii, Enterobacter cloacae ssp. cloacae, Serratia marcescens, Staphylococcus haemolyticus, and Staphylococcus epidermidis. The research result showed that 97.8\% MDR cases were reported in the NICU ward. Antibiotics which were still potent for all bacteria found in NICU patients were tigecycline, meropenem and ciprofloxacin (for Gram-negative bacteria) and tigecycline, linezolid, nitrofurantoin, moxifloksacin, and vancomycin (for Gram-positive bacteria).

Conclusion: A high percentage of MDR occurred in NICU patients. Sepsis is the most common diagnosis in NICU patients. The usage of third generation antibiotics should be limited and regulated systematically.

Keywords: Multi drug-resistance, Antibiotics, Neonatal intensive care unit.

(c) 2017 The Authors. Published by Innovare Academic Sciences Pvt Ltd. This is an open access article under the CC BY license (http://creativecommons. org/licenses/by/4. 0/) DOI: http://dx.doi.org/10.22159/ajpcr.2017.v10s2.19495

\section{INTRODUCTION}

Antibiotic resistance is a global issue today, which results in prolonged illness, disability and death. The World Health Organization (WHO) reported that antibiotic resistance has hit the world due to genetic change of bacteria, misuse of antibiotics for livestock and fishery products, and usage of antibiotics in non-infection diseases. Resistance of Klebsiella pneumoniae to carbapenem antibiotic has spread globally. $K$. pneumoniae bring about nosocomial infection in pneumonia, bloodstream infection (BSI), and infections in newborn babies treated in neonatal intensive care unit (NICU).

Methicillin-resistant Staphylococcus aureus (MRSA) has been found in communities and hospitals. WHO encourages coordinated efforts from all stakeholders to minimize the spread of antibiotic resistance [1]. Antibiotic resistance has also spread to Asian countries. In Nepal hospitals, antibiotic resistance is dominated by Gram-negative bacteria. These bacteria are resistant to quinolone and sulfonamide [2]. In Southern India, multidrug-resistance (MDR) incidences increase by $20 \%$, particularly Gram-negative bacteria, which are resistant to carbapenem [3]. In Indonesia, antibiotic resistance is not different from that of other Asian countries. Resistance due to Gram-negative bacteria has been reported by 10 teaching hospitals in Indonesia. One of them is perinatology, which occurred in a patient's ward in RSUP, Sanglah Bali.

Antibiotic resistance in patients of sepsis neonates is caused by Serratia marcescens [4]. Intensive care units, including NICU, are the most sensitive sections in a hospital because that is where patients with complication or the equipment used to treat life-threatening injuries are kept. Infections in NICU are generally caused by healthcare-associated infections (HAIs). HAIs are believed to be the direct and indirect cause of increases in morbidity, mortality and economic burden [5]. Bloodstream infections (BSIs) caused by commensal species are one of the infection types most commonly found in NICU (59\%) and it is life threatening [6].

Bacteria pattern and antibiotic sensitivity vary among countries and hospitals. Even in a single hospital, bacteria pattern vary among wards. Bacteria pattern variation also occurs across time. Since the 1950s, Staphylococcus aureus, Gram-negative bacteria and coagulase-negative staphylococci (CoNS) are the predominant causes of nosocomial infections in NICU in American hospitals. Today, Gram-positives, including MRSA, CoNS and vancomycin-resistant enterococci are MDR bacteria [7]. The same MDR was also reported to occur in NICUs in Australian, North American, European, and even Brazilian hospitals in the 2000s [8-10]. Therefore, the study's aims are to identify patterns of MDR in NICU in Central Java Hospital within the period of January 2014 to December 2015.

\section{METHODS}

\section{Study population}

Sample inclusion criteria

All neonatal patients in the NICU ward who had infections due to bacteria and on whom antibiotic sensitivity culture examination was performed.

\section{Sample exclusion criteria}

Neonatal patients in the NICU ward who had infections and have incomplete medical records. The protocol was approved by Medical and 
Health Research Ethics Committee, Faculty of Medicine, Gadjah Mada University (KE/FK/253/EC/2016).

\section{Patient evaluation}

The collected data of patients included characteristics of research subjects, that is, age, gender, birth history, diagnosis, length of stay (LoS), clinical outcome, and type of bacteria found.

\section{Antimicrobial susceptibility test}

This study was a descriptive study of data collected from a laboratory record of NICU in a hospital in Central Java. The collected data were all culture and sensitivity results using blood specimen. It was conducted within the period of January 2014-December 2015. Blood culture sensitivity examination was performed following the disc diffusion method as well as with agar dilution minimal inhibitory concentration method, using Vitek 2 (bioMérieux).

\section{Statistical analysis}

Research characteristic data, that is, gender, age, birth history, diagnosis, sepsis classification, LoS, clinical outcome, type of bacteria found, MDR, and bacterial resistance pattern against antibiotics were analyzed descriptively.

\section{RESULTS}

During the research period from January 2014 to December 2015, 225 neonatal patients were treated in the NICU of Central Java hospital. A 135 (60\%) patients died (Table 1).

The most common diagnosis of neonatal patients treated in NICU was sepsis infection, which was confirmed by patients' blood cultures. The neonatal characteristics are shown in Table 1. In general, the reason for the patients being admitted was infection and having baby birth weight below 1500 g. 90 neonatal patients (40\%) had sepsis infection along with other infections.

Microorganisms identified in patients' blood cultures are presented in Table 2. The most commonly identified microorganism was Gram-negative bacteria (72\%) which consisted of $K$. pneumoniae ssp. pneumoniae, Pseudomonas aeruginosa, Burkholderia cepacia, Acinetobacter baumannii, Enterobacter cloacae ssp. cloacae, and S. marcescens. Identified Gram-positive bacteria were Staphylococcus haemolyticus and Staphylococcus epidermidis. All neonatal patients used peripheral line cannulation, which most likely played a role in patients' LoS in NICU. Most of the neonates (85\%) were treated for over 7 days (Table 1 ).

Antibiotic resistance data of Gram-negative bacteria can be seen in Table 3. K. pneumoniae ssp. pneumoniae showed high resistance against penicillin and cephalosporin, aminoglycoside, as well as cotrimoxasole and piperacillin/tazobactam. Similar pattern was found in A. baumannii. $P$. aeruginosa showed a high resistance against all anti-pseudomonas antibiotics (25-100\%). B. cepacia showed moderate resistance against ceftazidime and levofloxacin $(<20 \%)$. E. cloacae ssp. cloacae showed resistance against penicillin and some cephalosporin. S. marcescens was resistant to tobramycin. Gram-positive bacteria, S. haemolyticus and $S$. epidermidis, have similar resistance pattern against penicillin, erythromycin, aminoglycoside, fluoroquinolone, clindamycin, rifampicin, and cotrimoxazole.

Antibiotic resistance data of Gram-positive bacteria can be seen in Table 4. Like S. epidermidis, S. haemolyticus showed high resistance against benzylpenicillin, erythromycin, clindamycin, ciprofloxacin, levofloxacin and rifampicin, gentamicin and cotrimoxazole (20-100\%). S. haemolyticus showed moderate resistance against moxifloxacin, nitrofurantoin, and vancomycin $(<20 \%)$. However, S. epidermidis showed high resistance against vancomycin $(25 \%)$.

The choice of sensitive-antibiotics for Gram-negative and Gram-positive microorganisms was presented in Table 5 .
Table 1: General characteristics of the admitted neonates $(n=225)$ during 1 -year period

\begin{tabular}{ll}
\hline Characteristics & $\mathbf{n = 2 2 5}(\mathbf{\%})$ \\
\hline Gender & \\
$\quad$ Male & $115(51.1)$ \\
$\quad$ Female & $110(48.9)$ \\
Age & \\
$\quad \leq 7$ days old & $138(61.3)$ \\
$\quad>7$ days old & $87(38.7)$ \\
Birthweight (g) & \\
$\quad<1500$ & $40(17.8)$ \\
$\quad 1500-2499$ & $120(53.3)$ \\
$\quad \geq 2000$ & $65(28.9)$ \\
Diagnosis & $135(60)$ \\
$\quad$ Sepsis & $90(40)$ \\
Sepsis and other infections & \\
Sepsis classification & $158(70.2)$ \\
$\quad$ EONS & $67(29.8)$ \\
$\quad$ LONS & \\
Length of stay & $140(62.2)$ \\
$\quad \leq 7$ days & $85(37.8)$ \\
$>7$ days & \\
Discharge status & $90(40)$ \\
Alive & $135(60)$ \\
$\quad$ Died & \\
\hline EONS: Early onset neonatal sepsis, LONS: Late onset neonatal sepsis
\end{tabular}

Table 2: The identified microorganisms in blood culture

\begin{tabular}{ll}
\hline Microorganism & $\mathbf{n = 2 2 5}(\mathbf{\%})$ \\
\hline Gram-negative organisms & \\
K. pneumoniae ssp. pneumoniae & $49(21.8)$ \\
P. aeruginosa & $31(13.8)$ \\
B. cepacia & $21(9.3)$ \\
A. baumannii & $21(9.3)$ \\
E. cloacae ssp. cloacae & $20(8.9)$ \\
S. marcescens & $20(8.9)$ \\
Gram-positive organisms & \\
S. haemolyticus & $32(14.2)$ \\
S. epidermidis & $31(13.8)$ \\
MDR & $220(97.8)$ \\
Non-MDR & $5(2.2)$ \\
\hline
\end{tabular}

K. pneumoniae ssp. pneumoniae: Klebsiella pneumoniae ssp. pneumoniae, P. aeruginosa: Pseudomonas aeruginosa, B. cepacia: Burkholderia cepacia A. baumannii: Acinetobacter baumannii, E. cloacae ssp. Cloacae: Enterobacter cloacae ssp. cloacae, S. marcescens: Serratia marcescens, S. haemolyticus: Staphylococcus haemolyticus, S. epidermidis: Staphylococcus epidermidis, MDR: Multidrug-resistance

\section{DISCUSSION}

MDR caused by nosocomial infection has drawn a lot of interest from people across the globe, especially since the neonatal patients are highly vulnerable to infections during their stay in the hospital. Neonatal infections are believed to be the biggest contributor to mortality and morbidity worldwide. In the WHO report, it was observed that about 4 million neonatal mortalities across the world occur due to infections, and about 1 million of them occur due to sepsis or pneumonia [11]. In this study, 225 neonatal patients admitted in NICU had BSIs. In developed countries, most nosocomial infections in neonatal patients are caused by BSIs and pneumonia [12], while in developing countries, most of the baby deliveries are performed at home, so various infections might occur, such as septicaemia, meningitis, respiratory infections, diarrhea and neonatal tetanus, although, BSIs poses a greater threat [13]. Neonatal sepsis incidents in Asia occur within the rate of 7.1-38/1000 live births. The most common bacteria causing neonatal sepsis in Asian and African countries are Gram-negative bacteria [2-4,13]. The result of this study also revealed that BSIs in NICU was dominated by Gramnegative bacteria ( $72 \%$ or 162 isolates out of 225 isolates). Around 
Table 3: Antibiotic resistance of Gram-negative organisms

\begin{tabular}{|c|c|c|c|c|c|}
\hline Antibiotics & $\begin{array}{l}\text { K. pneumonia ssp. } \\
\text { pneumoniae }(\%)\end{array}$ & P. aeruginosa (\%) & A. baumannii (\%) & E. cloacae ssp. cloacae $(\%)$ & S. marcescens (\%) \\
\hline \multicolumn{6}{|l|}{ Penicillin } \\
\hline Ampicillin & 100 & & 50 & 100 & \\
\hline Ampicillin/Sulbactam & 93.1 & & & 100 & \\
\hline \multicolumn{6}{|l|}{ Cephalosporin } \\
\hline Cefazolin & 100 & & & 100 & \\
\hline Cefoxitin & & & & 100 & \\
\hline Cefotaxime & 0 & & 100 & 0 & \\
\hline Ceftazidime & 93.3 & 50 & 80 & 12.5 & \\
\hline Ceftriaxone & 100 & & 20 & 12.5 & \\
\hline Cefepime & 93.3 & 57.2 & 50 & 7.1 & \\
\hline \multicolumn{6}{|l|}{ Carbapenem } \\
\hline Ertapenem & 0 & & & & 0 \\
\hline Imipenem & 0 & 50 & 0 & 0 & 0 \\
\hline Meropenem & & 25 & 0 & 6.2 & \\
\hline Ciprofloxacin & 0 & 33.3 & 20 & 14.3 & \\
\hline Levofloxacin & 0 & 50 & 50 & 25 & 0 \\
\hline \multicolumn{6}{|l|}{ Aminoglycoside } \\
\hline Amikacin & 23.3 & 75 & 66.7 & 5.9 & 5.3 \\
\hline Gentamicin & 93.1 & 71.4 & 50 & 11.8 & 10.5 \\
\hline Tobramycin & & 100 & 50 & 0 & 100 \\
\hline \multicolumn{6}{|l|}{ Tetracycline } \\
\hline Tetracycline & & & 100 & & \\
\hline Tigecycline & 0 & & 0 & & \\
\hline \multicolumn{6}{|l|}{ Sulfa } \\
\hline Cotrimoxazole & 66.7 & & 20 & 21.4 & \\
\hline Piperacillin/Tazobactam & 85.2 & & & & 0 \\
\hline Fosfomycin & 0 & & & & \\
\hline \multicolumn{6}{|l|}{ Amphenicol } \\
\hline Chloramphenicol & 0 & & & & \\
\hline
\end{tabular}

K. pneumoniae ssp. pneumoniae: Klebsiella pneumoniae ssp. pneumoniae, P. aeruginosa: Pseudomonas aeruginosa, A. baumannii: Acinetobacter baumannii, E. cloacae ssp. Cloacae: Enterobacter cloacae ssp. cloacae, S. marcescens: Serratia marcescens

Table 4: Antibiotic resistance of Gram-positive organisms

\begin{tabular}{lll}
\hline Antibiotics & S. haemolyticus $\mathbf{( \% )}$ & S. epidermidis $\mathbf{~ \% )}$ \\
\hline $\begin{array}{l}\text { Penicillin } \\
\text { Benzylpenicillin }\end{array}$ & 96.7 & 100 \\
$\begin{array}{l}\text { Macrolide } \\
\text { Erythromycin }\end{array}$ & 80.6 & \\
$\begin{array}{l}\text { Aminoglycoside } \\
\text { Amikacin }\end{array}$ & & 81.2 \\
Gentamicin & 67.7 & 0 \\
Fluoroquinolon & & 74.2 \\
Ciprofloxacin & 41.9 & \\
Levofloxacin & 35.5 & 70.9 \\
Moxifloxacin & 3.2 & 70.9 \\
Tetracycline & & 16.1 \\
Tetracycline & 9.7 & \\
Tigecycline & 0 & 32.3 \\
Clindamycin & 87.1 & 0 \\
Linezolid & 0 & 93.7 \\
Nitrofurantoin & 3.2 & 0 \\
Rifampicin & 25.8 & 3.2 \\
Sulfa & & 77.4 \\
Cotrimoxazole & 22.6 & 21.9 \\
Vancomycin & 10 & 25 \\
\hline
\end{tabular}

$35 \%$ dominant bacteria were $K$. pneumoniae ssp. pneumoniae and P. aeruginosa. The research result was in line with the bacteria patterns found to be sources of infections in patients treated in NICU in Suez Canal University Hospital, Ismalia, Egypt, and other surveillances in Egypt $[14,15]$. Klebsiella sp. has for long been known as the cause of sepsis, urinary tract infection and pneumonia in NICU. In the 1970s, there was an outbreak in NICU due to kanamycin-resistant Klebsiella sp. Furthermore, extended-spectrum beta-lactamase-
Table 5: Sensitive-antibiotic choice for the microorganisms

\begin{tabular}{|c|c|}
\hline Organism & Sensitive-antibiotic \\
\hline \multicolumn{2}{|l|}{ Gram-negative } \\
\hline $\begin{array}{l}\text { K. pneumoniae ssp. } \\
\text { pneumoniae }\end{array}$ & $\begin{array}{l}\text { Cefotaxime, ertapenem, imipenem, } \\
\text { ciprofloxacin, levofloxacin, tigecycline, } \\
\text { fosfomycin }\end{array}$ \\
\hline P. aeruginosa & None \\
\hline B. серасіа & Cotrimoxazole, levofloxacin, ceftazidime \\
\hline A. baumannii & $\begin{array}{l}\text { Imipenem, meropenem, tigecycline, } \\
\text { ceftriaxone, ciprofloxacin, cotrimoxazole }\end{array}$ \\
\hline E. cloacae ssp. cloacae & $\begin{array}{l}\text { Cefotaxime, ceftriaxone, cefuroxime, } \\
\text { cefepime, imipenem, meropenem, } \\
\text { ciprofloxacin, gentamicin, amikacin, } \\
\text { tobramycin }\end{array}$ \\
\hline S. marcescens & $\begin{array}{l}\text { Ertapenem, imipenem, levofloxacin, } \\
\text { piperacillin/tazobactam }\end{array}$ \\
\hline \multicolumn{2}{|l|}{ Gram-positive } \\
\hline S. haemolyticus & $\begin{array}{l}\text { Moxifloxacin, tigecycline, linezolid, } \\
\text { nitrofurantoin, vancomycin }\end{array}$ \\
\hline S. epidermidis & $\begin{array}{l}\text { Moxifloxacin, tigecycline, amikacin, } \\
\text { linezolid, nitrofurantoin }\end{array}$ \\
\hline
\end{tabular}

K. pneumoniae ssp. pneumoniae: Klebsiella pneumoniae ssp. pneumoniae, P. aeruginosa: Pseudomonas aeruginosa, B. cepacia: Burkholderia cepacia, A. baumannii: Acinetobacter baumannii, E. cloacae ssp. cloacae: Enterobacter cloacae ssp. cloacae, S. marcescens: Serratia marcescens, S. haemolyticus: Staphylococcus haemolyticus, S. epidermidis: Staphylococcus epidermidis

producing (ESBL) Klebsiella sp. is now found to be resistant to thirdgeneration cephalosporins [6]. The study performed in several clinics in Nepal also found the ESBL Klebsiella sp. to be resistant to third generation cephalosporin. Incidences of ESBL Klebsiella sp. which 
was found to be resistant to cephalexin and ceftriaxone are 14.29 and $3.5 \%$, respectively $[16,17]$.

The study by Lamichhane et al. (2014) revealed that MDR incidences in non ESBL Klebsiella sp. is also very high, being around 50\%. The bacteria show resistance to cephalexin and ceftriaxone [3]. Incidences of Klebsiella sp. resistance to ceftriaxone in the US is around $6.6 \%$ and in Latin America, it is much higher (around 30-50\%) [18]. This study also found Klebsiella sp. being resistant to third-generation cephalosporin (ceftriaxone and ceftazidime) with 100 and 93.3\% rates (respectively). In addition, the Klebsiella sp. identified in this study also showed resistance against aminoglycoside, cotrimoxazole and piperacillin/ tazobactam. It showed that the MDR in NICU at the Central Java hospital was alarming. Lack of study in Indonesia leads to a lack of national awareness of antibiotic resistance control, although it is very likely that MDR also occurs in various regions in Indonesia. There is even MDR variation in different units in a single hospital. In 2016, Indonesian government started a national surveillance on the qualitative and quantitative usage of antibiotics to get a picture of rationality of antibiotic usage in several teaching hospitals, to create a map of the influence of rationality of antibiotic prescription for MDR and national antibiotic control strategy.

Gram-negative bacteria are potent organisms which cause BSIs. Gram-negative bacteria are the more rapidly acquired MDR than the Gram-positive bacteria. Several studies reveal major threats due to Gram-negative bacteria, particularly to patients admitted in the hospital. The increasing usage of antibiotic carbapenem leads to carbapenem-resistant Gram-negative incidences in several countries $[18,19]$. Carbapenem is usually used for MDR A. baumannii which eventually causes carbapenem-resistant $A$. baumannii, commonly found in sputum and urine specimens [3]. MDR A. baumannii occur in patients with immunosuppression or serious illness. Furthermore, a prolonged stay in the intensive care unit, including NICU, also makes it easier for MDR A. baumannii, which comes from ventilation equipment and air conditioners [20,21]. This study found A. baumannii to be resistant to penicillin, cephalosporin, fluoroquinolone, aminoglycoside, tetracycline, and cotrimoxazole but it is still being sensitive to carbapenem.

Another dangerous Gram-negative bacteria are $P$. aeruginosa. $P$. aeruginosa infection is known to be death sources in sepsis and pneumonia. P. aeruginosa can be as a result of transmission from resuscitation equipment, humidifiers, incubator, and other equipment in NICU. Another source of transmission of $P$. aeruginosa is the hands of health-care workers [5]. The incidence of $P$. aeruginosa resistance in one of Tamil Nadu hospitals in India is over $50 \%$ for all $\beta$-lactam antibiotics, aminoglycoside, and fluoroquinolone [22]. $P$. aeruginosa identified in NICU in the present study showed resistance against all $\beta$-lactam antibiotics, aminoglycoside, and fluoroquinolone, as well as carbapenem at the rate of $25-100 \%$. There was practically no anti-pseudomonas antibiotic left for therapy in NICU.

S. epidermidis and S. hemoliticus are part of CoNS. CoNS isolate showed that MDR resulted from its ability to form biofilm. Resistance against CoNS, similar to other bacteria, can be caused by overuse of antibiotics. In patients admitted in NICU, CoNS can form biofilm in the form of colonization, worsened by the severity of the disease suffered by preterm neonatal patients [23]. Nosocomial infections in Australia, North America, and Europe are mostly caused by CoNS with 48-75\% incidences [8-10]. Although nosocomial infection incidence by CoNS is high, it rarely leads to death. From the study in NICU at the central Java hospital, only antibiotics tigecycline and linezolid were found not to be resistant to CoNS bacteria.

\section{CONCLUSION}

Considering the rate of MDR in the NICU in one of the hospitals in central Java, there should be concerted efforts by the hospital, coordinated by the antibiotic resistance control team to reduce MDR rate, especially in NICU, and to improve patients' therapy outcome. The usage of third generation antibiotics should be limited and regulated systematically.

\section{REFERENCES}

1. Online Archive of World Health Organization. Antimicrobial Resistance. Available from: http://www.who.int. [Last cited on 2016 Oct 17].

2. Lamichhane B, Thakur C, Jain SK. Antibiotic resistance patterns of Gram-negative isolates in a tertiary care hospital of nepal. Asian $\mathrm{J}$ Pharm Clin Res 2014;7(3):30-3.

3. Natarajan SD, Viswajith V, Kandhasamy M, Ravichandran M, Munisamy P, Chandrasekar V. An epidemiological study on deadly drug-defying untreatable carbapenem-resistant Gram-negative bacteria encountered in South India. Asian J Pharm Clin Res 2015;8(6):63-7.

4. Kardana IM. Study of organisms and antibiotic sensitivity in perinatology unit. Sari Pediatri 2011;12(6):381-5.

5. Payne NR, Carpenter JH, Badger GJ, Horbar JD, Rogowski J. Marginal increase in cost and excess length of stay associated with nosocomial bloodstream infections in surviving very low birth weight infants. Pediatrics 2004;114(2):348-55.

6. Polin RA, Saiman L. Nosocomial infections in the neonatal intensive care unit. Neoreviews 2003;4(3):e81-8.

7. Ho TS, Wang SM, Wu YH, Shen $\mathrm{CF}$, Lin $\mathrm{YJ}$, Lin $\mathrm{CH}$, et al. Long-term characteristics of healthcare-associated infections in a neonatal intensive care unit. J Microbiol Immunol Infect 2010;43(5):407-15

8. Kawagoe JY, Segre CA, Pereira CR, Cardoso MF, Silva CV, Fukushima JT. Risk factors for nosocomial infections in critically ill newborns: A 5-year prospective cohort study. Am J Infect Control 2001;29(2):109-14.

9. Urrea M, Iriondo M, Thio M, Krauel X, Serra M, LaTorre C, et al. A prospective incidence study of nosocomial infections in a neonatal care unit. Am J Infect Control 2003;31(8):505-7.

10. Isaacs D. A ten year, multicentre study of coagulase negative staphylococcal infections in Australasians neonatal units. Arch Dis Child Fetal Neonatal Ed 2003;88(2):F89-93.

11. Online Archive of World Health Organization. Neonatal Sepsis - A Major Killer to be Tackled in Communities. Available from: http:// www.who.int. [Last cited on 2016 Oct 17].

12. Hyde TB, Hilger TM, Reingold A, Farley MM, O’Brien KL, Schuchat A; Active Bacterial Core surveillance (ABCs) of the Emerging Infections Program Network. Trends in incidence and antimicrobial resistance of early-onset sepsis: Population-based surveillance in San Francisco and Atlanta. Pediatrics 2002;110(4):690-5.

13. Vergnano S, Sharland M, Kazembe P, Mwansambo C, Heath PT. Neonatal sepsis: An international perspective. Arch Dis Child Fetal Neonatal Ed 2005;90(3):F220-4.

14. Abdel-Wahab F, Ghoneim M, Khashaba M, El-Gilany AH, Abdel-Hady D. Nosocomial infection surveillance in an Egyptian neonatal intensive care unit. J Hosp Infect 2013;83(3):196-9.

15. Kishk RM, Mandour MF, Farghaly RM, Ibrahim A, Nemr NA. Pattern of blood stream infections within neonatal intensive care unit, suez canal university hospital, Ismailia, Egypt. Int J Microbiol 2014;2014:276873.

16. Kumarasamy KK, Toleman MA, Walsh TR, Bagaria J, Butt F, Balakrishnan $\mathrm{R}$, et al. Emergence of a new antibiotic resistance mechanism in India, Pakistan, and the UK: A molecular, biological, and epidemiological study. Lancet Infect Dis 2010;10(9):597-602.

17. Upadhyay AK, Parajuli P. Extended spectrum $\beta$-lactamase producing multidrug resistant Klebsiella species isolated at National Medicine College and Teaching hospital, Nepal. Asia J Pharm Clin Res 2013;6(4):161-4

18. Sader HS, Jones RN, Gales AC, Winokur P, Kugler KC, Pfaller MA, et al. Antimicrobial susceptibility pattern for pathogens isolated from patient in Latin American medical centres with a diagnosis of pneumonia analysis results from the Sentry antimicrobial surveillance program (1997) Sentry Latin American Study Group. Diagn Microbiol Infect Dis 1998;32(4):289-301.

19. Panta K, Ghimire P, Rai SK, Mukhiya RK, Singh RN, Rai G. Antibiogram typing of Gram-negative isolates in diferent clinical samples of a tertiary hospital. Asian J Pharm Clin Res 2013;6(1):153-6. 
20. Tabassum S. Multidrug-resistant (MDR) Acinetobacter: A major nosocomial pathogen challenging physicians. Bangladesh J Med Microbiol 2007;1(2):65-8.

21. Villegas MV, Hartstein AI. Acinetobacter outbreaks, 1977-2000. Infect Control Hosp Epidemiol 2003;24(4):284-95.

22. Senthamarai S, Reddy SK, Sivasankari S, Anitha C, Somasunder V, Kumudhavathi MS, et al. Resistance pattern of Pseudomonas aeruginosa in a Tertiary Care Hospital of Kanchipuram, Tamilnadu, India. J Clin Diagn Res 2014;8(5):DC30-2.

23. Foka A, Chini V, Petinaki E, Kolonitsiou F, Anastassiou ED, Dimitracopoulos G, et al. Clonality of slime-producing methicillinresistant coagulase-negative staphylococci disseminated in the neonatal intensive care unit of a university hospital. Clin Microbiol Infect 2006;12(12):1230-3. 